\title{
Supporting Information \\ Clathrate Hydrate Inhibition by Polyisocyanate with Diethylammonium Group
}

In Gyu Bak, ${ }^{\dagger}$ Chi-Ho Heo, ${ }^{\dagger}$ Malcolm A. Kelland, ${ }^{\dagger}$ Eunji Lee,${ }^{\dagger}$ Beom-Goo Kang, ${ }^{\S}, *$ and Jae-Suk Lee ${ }^{\dagger, *}$ 'School of Materials Science and Engineering, Gwangju Institute of Science and Technology (GIST), 123 Cheomdangwagiro, Buk-gu, Gwangju 61005, Korea

tDepartment of Chemistry, Bioscience and Environmental Engineering, University of Stavanger, N-4036 Stavanger, Norway

${ }^{\S}$ Department of Chemical Engineering, Soongsil University, Seoul 06978, Korea

§,*E-mail: bkang@ssu.ac.kr, Tel: (+82)-2-820-0615

†,*E-mail: jslee@gist.ac.kr; Tel: (+82)-62-715-2306 

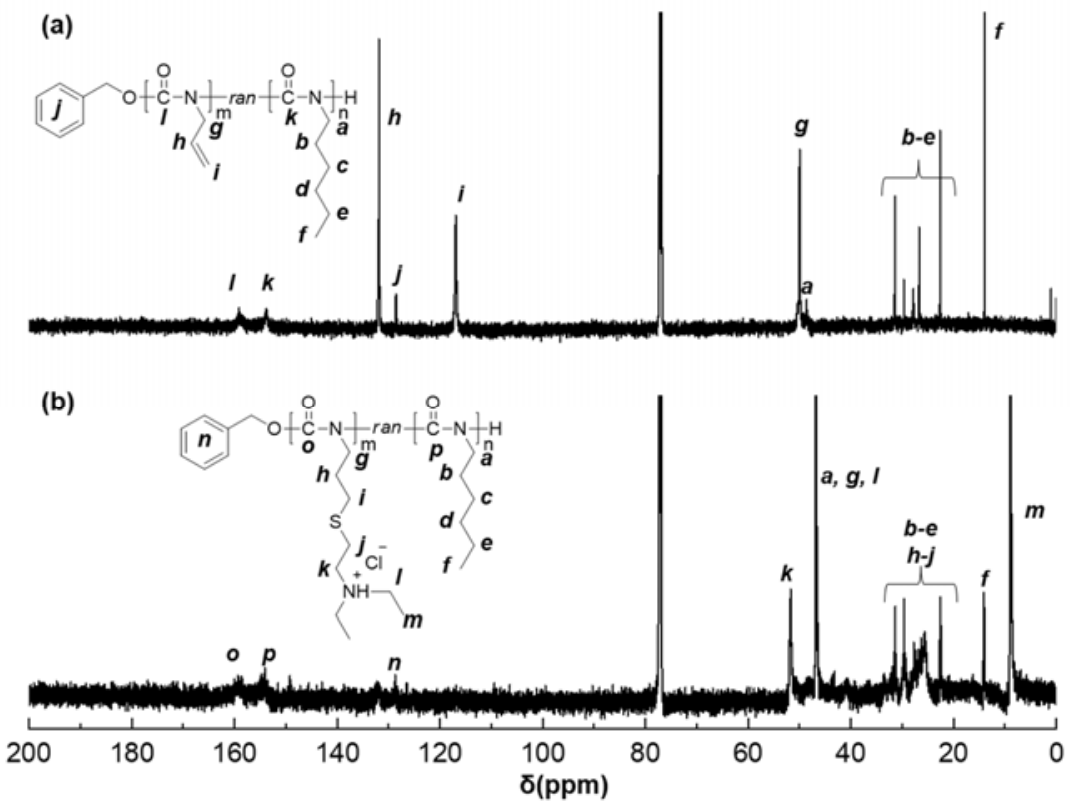

Figure S1. ${ }^{13}$ C NMR spectra of (a) P(AIC-ran-HIC) and (b) P(DETPIC-ran-HIC).
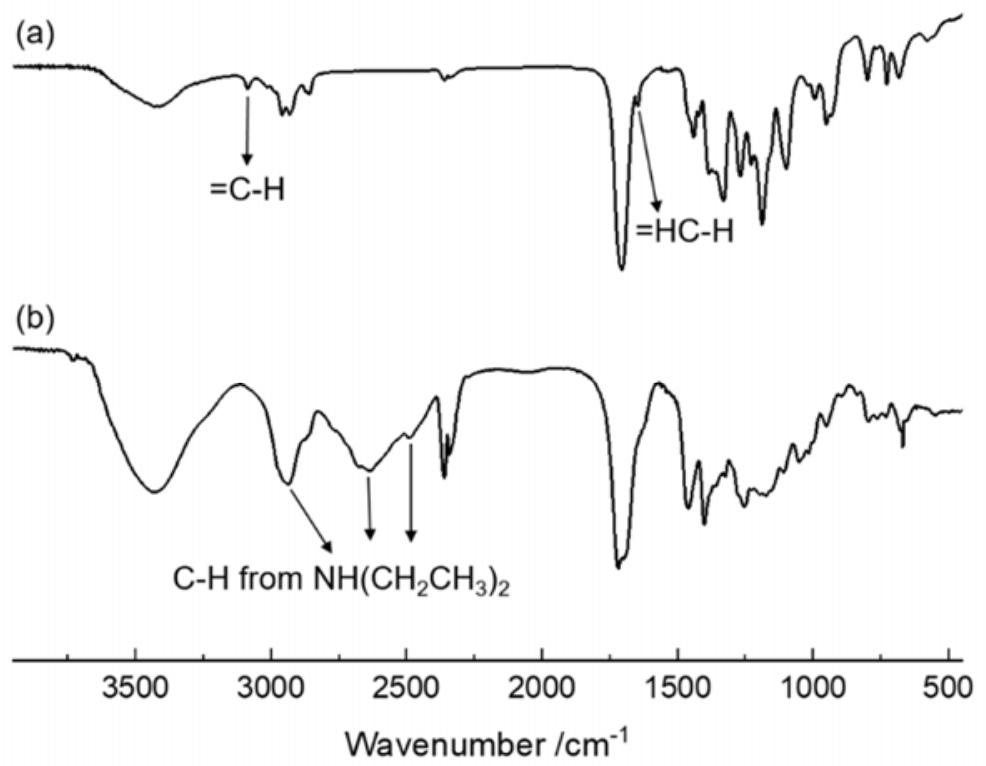

Figure S2. FT-IR spectra of (a) P(AIC-ran-HIC) and (b) P(DETPIC-ran-HIC). 